\title{
ACTPol: on-sky performance and characterization
}

E. Grace ${ }^{a}$, J. Beall ${ }^{b}$, J. R. Bond ${ }^{c}$, H.M. Cho ${ }^{b}$, R. Datta ${ }^{j}$, M. J. Devlin ${ }^{d}$, R. Dünner ${ }^{e}$,

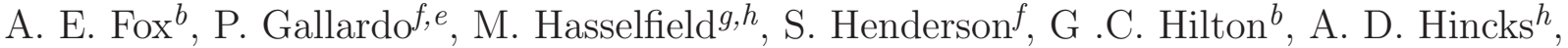

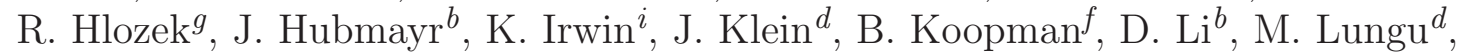
L. Newburgh ${ }^{a}$, J. P. Nibarger ${ }^{b}$, M. D. Niemack ${ }^{f}$, L. Maurin ${ }^{e}$, J. McMahon $^{j}$, S. Naess ${ }^{k}$,

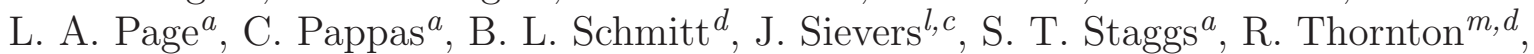
J. Van Lanen ${ }^{b}$, E.J. Wollack ${ }^{m}$

${ }^{a}$ Joseph Henry Laboratories of Physics, Jadwin Hall, Princeton University, Princeton, NJ, 08544;

${ }^{b}$ NIST Quantum Devices Group, 325 Broadway Mailcode 817.03, Boulder, CO, USA 80305; ${ }^{c}$ Canadian Institute for Theoretical Astrophysics, University of Toronto, Toronto, ON, Canada M5S 3H8;

${ }^{d}$ Department of Physics and Astronomy, University of Pennsylvania, 209 South 33rd Street, Philadelphia, PA, USA 19104;

${ }^{e}$ Departamento de Astronomía y Astrofísica, Pontificía Universidad Católica,Casilla 306, Santiago 22, Chile;

${ }^{f}$ Department of Physics, Cornell University, Ithaca, NY, USA 14853;

${ }^{g}$ Department of Astrophysical Sciences, Peyton Hall, Princeton University, Princeton, NJ USA 08544

${ }^{h}$ Department of Physics and Astronomy, University of British Columbia, Vancouver, BC, Canada V6T 1Z4;

${ }^{i}$ Department of Physics, Stanford University, Stanford, CA, USA 94305-4085;

${ }^{j}$ Department of Physics, University of Michigan, Ann Arbor, USA 48103;

${ }^{k}$ Sub-Department of Astrophysics, University of Oxford, Keble Road, Oxford, UK OX1 3RH; ${ }^{l}$ Astrophysics and Cosmology Research Unit, School of Chemistry and Physics, University of

KwaZulu-Natal, Durban 4041, South Africa;

${ }^{m}$ Department of Physics, West Chester University of Pennsylvania, West Chester, PA, USA 19383

${ }^{n}$ NASA/Goddard Space Flight Center, Greenbelt, MD, USA 20771

\begin{abstract}
ACTPol is the polarization-sensitive receiver on the Atacama Cosmology Telescope. ACTPol enables sensitive millimeter wavelength measurements of the temperature and polarization anisotropies of the Cosmic Microwave Background $(\mathrm{CMB})$ at arcminute angular scales. These measurements are designed to explore the process of cosmic structure formation, constrain or determine the sum of the neutrino masses, probe dark energy, and provide a foundation for a host of other cosmological tests. We present an overview of the first season of ACTPol observations focusing on the optimization and calibration of the first detector array as well as detailing the on-sky performance.
\end{abstract}

Keywords: Cosmology, Cosmic Microwave Background, Polarimetry, Transition Edge Sensor, Bolometer

Further author information: (Send correspondence to E. Grace)

E. Grace.: E-mail: egrace@princeton.edu

Millimeter, Submillimeter, and Far-Infrared Detectors and Instrumentation for Astronomy VII,

edited by Wayne S. Holland, Jonas Zmuidzinas, Proc. of SPIE Vol. 9153, 915310 - @ 2014

SPIE · CCC code: $0277-786 X / 14 / \$ 18 \cdot$ doi: $10.1117 / 12.2057243$

Proc. of SPIE Vol. 9153 915310-1 


\section{INTRODUCTION}

Over the past two decades, observations of the Cosmic Microwave Background (CMB) have been invaluable for advancing our knowledge of cosmology. These measurements have played a pivotal role in establishing the $\Lambda \mathrm{CDM}$ model as the standard model of cosmology and providing high precision measurements of the model parameters. The temperature anisotropies have been well characterized by a large number of experiments including most recently ACT, ${ }^{1}$ Planck, ${ }^{2}$ SPT, ${ }^{3}$ and WMAP. ${ }^{4}$ The polarization of the CMB provides an additional probe of cosmology, both as a complement to the temperature anisotropy measurements and as a unique measure of new physical phenomena. ${ }^{5}$

Aiming to take advantage of this rich signal, the ACTPol receiver, a polarization-sensitive upgrade to the Atacama Cosmology Telescope (ACT), was deployed in the summer of 2013. This paper describes the design, assembly, and performance of the first ACTPol detector array, which achieved first light in July of 2013. There followed three months of science observations in 2013 resulting in a precision measurement of the E-mode spectrum at $\ell>200$ as described in Naess et al $2014 .^{6}$

\section{INSTRUMENT SUMMARY}

ACT is located on Cerro Toco at an elevation of 5200 meters in Chile, a location which benefits from the high elevation, the dry conditions, the opportunity for cross-linking observations, and overlap with other multifrequency observations. The first generation ACT camera (the Millimeter Bolometer Array Camera or MBAC) observed the temperature anisotropies of the CMB with $1.3^{\prime}$ resolution from 2008 to $2010 .{ }^{7}$ The ACTPol upgrade continues to use the original ACT mirrors, a 6 meter primary and a 2 meter secondary in an off-axis Gregorian configuration, but introduces a new cryogenic receiver, which is designed to house three independent optics tubes. $^{8}$ In each optics tube there are three cryogenic silicon lenses to reimage the Gregorian focus of the telescope. These lenses are anti-reflection coated using a meta-material technique in which grooves are cut in the silicon surface to simulate the desired index of refraction. ${ }^{9}$ The first of these three optics tubes, which was deployed in 2013, contains a detector array consisting of 1044 detectors sensitive to $146 \mathrm{GHz}$, known as PA1. We are deploying a second $146 \mathrm{GHz}$ array (PA2) and a third array (PA3) that employs dichroic detectors with simultaneous sensitivity to $90 \mathrm{GHz}$ and $146 \mathrm{GHz} .{ }^{10}$ For each array, the incident radiation is coupled onto the detectors via a monolithic feedhorn array assembled from a stack of silicon wafers, a design which enables precision corrugations, dense detector packing, and thermal matching to the silicon detector wafers. ${ }^{11}$ The upper edge of the detector bandpass is determined by Cardiff's free space metal mesh filters, ${ }^{12}$ while the lower edge is defined by the waveguide cutoff of the feed horns. The band center is $146 \pm 3 \mathrm{GHz}$ with a bandwidth of 49 GHz. ${ }^{6}$ Additionally, thermal blocking filters are used in each optics tube. ${ }^{13}$ A continuous dilution refrigerator provides the cooling for the detector arrays, achieving an operating array bath temperature of $80 \mathrm{mK}$.

\subsection{Detectors}

Each pixel in an ACTPol array is composed of an ortho-mode transducer (OMT) coupled to a pair of transition edge sensor (TES) bolometers. ${ }^{14}$ The planar OMT on each pixel is coupled to a waveguide input and a quarterwave backshort. ${ }^{15}$ Each pair of antenna probes on the OMT separate the incident radiation into two perpendicular polarization signals, ${ }^{16}$ which are then fed on niobium microstrip lines to a pair of TES islands where the power is deposited via a lossy gold meander. As the island heats relative to the bath temperature, the molybdenum-gold bilayer, which has been biased onto its superconducting transition, responds with a change in resistance. The ACTPol pixels are fabricated at the National Institute of Standards and Technology (NIST) on three-inch monolithic silicon wafers in two varieties - the semi-hexagonal wafer (semihex), housing 47 pixels (94 TESes), and the hexagonal wafer (hex), housing 127 pixels (254 TESes). A full ACTPol $146 \mathrm{GHz}$ array contains three hex and three semihex wafers for a total of 1044 TESes, of which 1014 are connected to the read-out circuitry due to the number of channels available. 


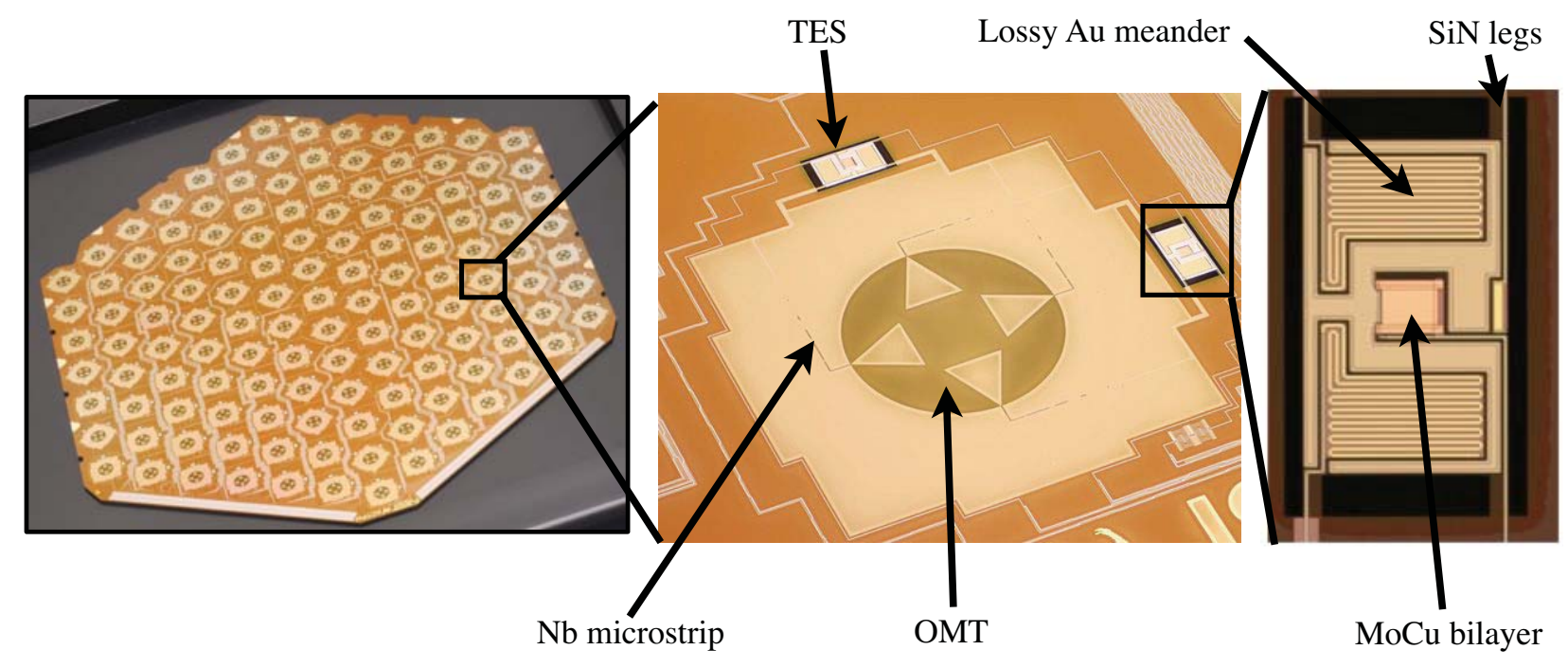

Figure 1: (Left) An ACTPol hex wafer containing 127 pixels each with two TESes. Each array contains three of these hexes and three semihex wafers, for a total of 522 pixels. The bond pads which connect the detector bias lines on the wafer to the rest of the circuit can be seen on the front edges. Each hex is fabricated on a 3 inch wafer. (Middle) A single ACTPol pixel. The incident radiation couples to each detector via the OMT in the middle of the pixel. From here the power is directed onto the niobium microstrip lines where it is carried onto one of the two TES islands on the edges. The pixels have $5 \mathrm{~mm}$ spacing. (Right) An ACTPol TES. The power is deposited on the island as heat through the lossy Au meander. The island is thermally isolated from the bath, connected only through four small SiN legs which hold the TES bias and signal lines. The increase in temperature of the island resulting from the deposited power is detected by the change in resistance of the $\mathrm{MoCu}$ bilayer which has been biased onto its superconducting transition. The TES island is $0.3 \mathrm{~mm}$ wide.

\begin{tabular}{|c|c|c|c|c|c|c|}
\hline Wafer & Wafer Type & Yield (\%) & $T_{c} \mathbf{( m K )}$ & $P_{\text {sat }}(\mathbf{p W})$ & $\mathbf{G}(\mathbf{p W} / \mathbf{K})$ & $\eta_{\text {det }} \mathbf{( \% )}$ \\
\hline \hline W10 & Hex & 71 & $143 \pm 5$ & $8.4 \pm 1.7$ & $226 \pm 30$ & $59 \pm 5$ \\
\hline W09 & Hex & 71 & $143 \pm 5$ & $8.5 \pm 1.6$ & $225 \pm 24$ & $61 \pm 8$ \\
\hline W08 & Hex & 81 & $146 \pm 6$ & $7.1 \pm 1.8$ & $173 \pm 33$ & $62 \pm 7$ \\
\hline SH1A & Semihex & 73 & $158 \pm 5$ & $14.4 \pm 1.9$ & $326 \pm 25$ & $47 \pm 14$ \\
\hline SH2A & Semihex & 80 & $156 \pm 6$ & $14.4 \pm 2.1$ & $327 \pm 24$ & $18 \pm 12$ \\
\hline SH2B & Semihex & 78 & $151 \pm 4$ & $12.3 \pm 1.2$ & $303 \pm 19$ & $21 \pm 12$ \\
\hline
\end{tabular}

Table 1: The measured average ACTPol detector parameter values and detector yield by wafer for the first $146 \mathrm{GHz}$ array as measured in the full receiver. here $P_{\text {sat }}$ is defined at $80 \mathrm{mK}$ in the absence of the optical loading and $\eta_{\text {det }}$ is a measure of the proportion of optical power incident on the detector which is actually detected by the TES.. This includes losses in the OMT and pixel microstrip lines and is measured by comparing an observed difference in loading with the predicted power difference assuming optimal detector coupling. The errors bars are the standard deviation of the detector distribution.

The design of the ACTPol TESes was driven by the need to optimize the detector performance and noise properties under the predicted bath temperature and optical loading. The saturation power $\left(P_{\text {sat }}\right)$, or the amount of loading needed to drive the TES normal, determines the range of conditions under which the detector can be operated. The saturation power is determined by the bath temperature $\left(T_{b}\right)$, the TES transition temperature $\left(T_{c}\right)$, and the thermal conductivity from the TES island to the bath $(G)$.

$$
P_{\text {sat }}=\kappa\left(T_{c}^{n}-T_{b}^{n}\right) .
$$



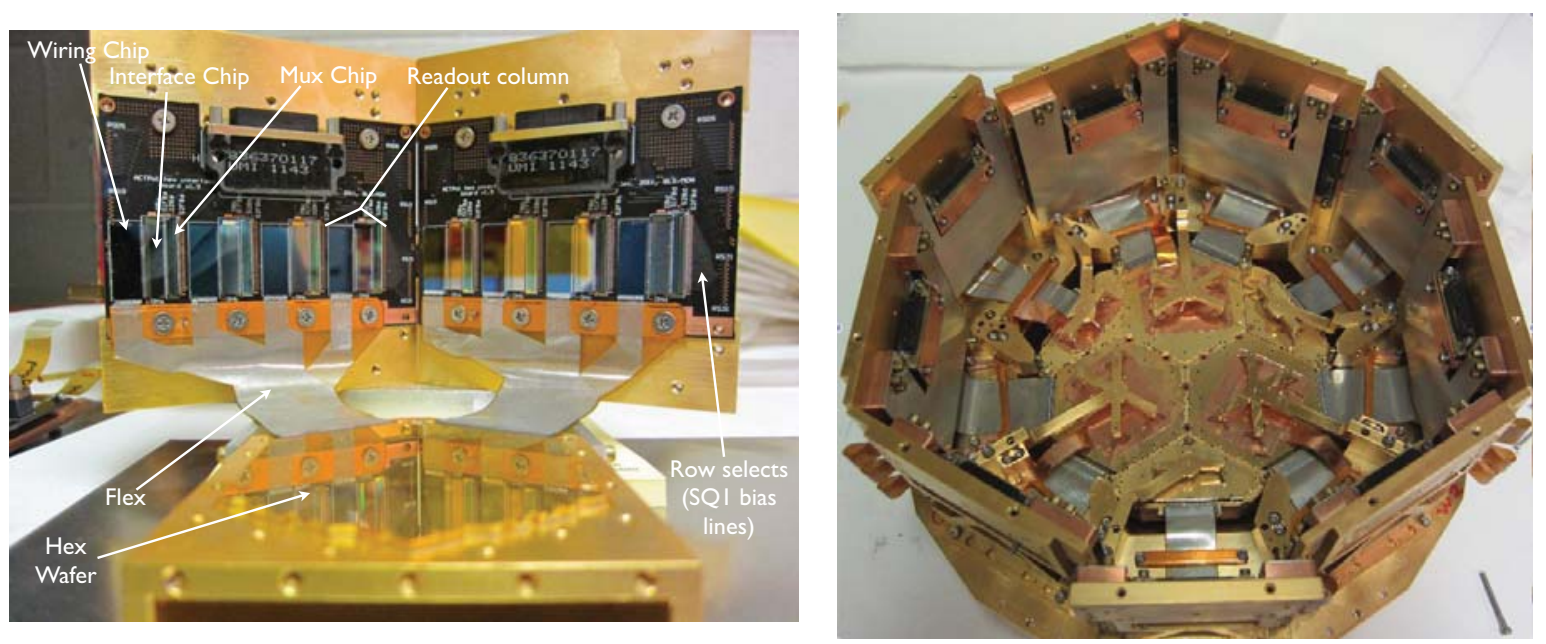

Figure 2: (Left) A fully assembled hex wafer viewed edge on. There are two hex PCBs each containing four readout columns. The columns are each composed of, from right to left, a mux chip, an interface chip, and a wiring chip. The row addressing SQ1 bias lines ("row selects") are bonded to the mux chips from the PCB bond pads on the right of the chip. The rest of the bias and feedback lines are connected to the PCB via the MDM connector seen at the top of each board. The detector bias lines are carried from the PCB to the wafer via the folded flex which is bonded at either end. (Right) The fully assumed PA1 array package. The six detector wafers sit in the middle while the readout PCBs are arrayed around the edge. In this photo, the view is from the back of the detector wafers with feedhorn apertures pointing down.

Here the variables $\kappa$ and $n$, which are determined by the geometry and material properties of the TES legs, parametrize the thermal conductivity to the bath.

$$
G=\frac{d P_{\mathrm{sat}}}{d T_{c}}=n \kappa T_{c}^{n-1} .
$$

For the PA1, the target $P_{\text {sat }}$ was $13.5 \mathrm{pW}$ at a bath temperature of $80 \mathrm{mK}$. This was selected with the goal to exceed the expected optical loading by a factor of three. The target transition temperature was chosen as $150 \mathrm{mK}$ and the detector leg width as $29.5 \mathrm{um}$ to optimize the detector thermal noise properties under the constraint of this chosen saturation power and bath temperature.

Another key detector property is the optical coupling efficiency $\left(\eta_{\text {det }}\right)$, which we define as the ratio of the measured power on the TES to the predicted in-band loading coupling to the feedhorns. It is measured by comparing an observed change in loading with the predicted change for a detector with zero-loss.

A summary of the achieved detector properties for PA1 as measured in pre-deployment testing can be see in Table 1.

\subsection{Detector Readout}

The detectors are read-out using three stages of superconducting quantum interference device (SQUID) amplification in a time domain multiplexing (TDM) scheme. ${ }^{17}$ This read-out has the advantage of limiting the thermal conductivity to the cold stage through the reduced number of wires. The current through each TES is coupled to its own first stage SQUID (SQ1) through an inductive coil. A set of 32 of these SQ1s are coupled to a single second stage SQUID (SQ2) through a summing coil. The SQ2 and SQ1s for a set of 32 detectors are housed on a single multiplexing chip ("MUX11c", developed by NIST) which is cooled to $80 \mathrm{mK}$. By biasing one SQ1 on each of the MUX chips at a time in rapid succession, each detector can be read out individually by its SQ2 stage. The third and final stage of amplification is accomplished by a set of SQUID series arrays (SA). The detectors are voltage biased onto their transition using an $\sim 180 \mu \Omega$ shunt resistor, housed on the Nyquist chip which also contains a $600 \mathrm{nH}$ inductor designed to band limit the response. The biasing and multiplexing is handled 
using the Multi-Channel Electronics (MCE) box developed at UBC. ${ }^{18}$ This readout system has a raw sampling rate of $50 \mathrm{MHz}$. Using a row-switching rate of $500 \mathrm{kHz}$ with 33 row selects, ACTPol achieves a $15.15 \mathrm{kHz}$ row sampling rate. This signal is then filtered using a four-pole Butterworth, low-pass, anti-aliasing filter with a $120 \mathrm{~Hz}$ cutoff. The data are then downsampled to $398.7 \mathrm{~Hz}$ and transmitted by fibre to the data acquisition computer for recording to disk.

\subsection{Array Assembly}

A fully assembled ACTPol wafer consists of three silicon layers - a waveguide interface plate (WIP) wafer designed to couple to the feed-horn stack, the detector wafer itself, and a back-short assembly which forms the quarter-wavelength cavity. Each wafer stack is placed directly onto the surface of the silicon feedhorn stack with alignment pins for precise placement and clamped from the back using 3 (5) BeCu tripod springs for the semihexes (hexes). The detector bias lines are carried onto the detector wafer through a wire-bonded connection to a piece of double-layered flexible circuity consisting of a Kapton base with aluminum traces at $100 \mathrm{um}$ pitch. This flex is wire-bonded on the other end to a printed circuit board (PCB) which holds the multiplexing and readout chips. In total there are nine of these boards, one for each semihex wafer and two for each hex, all operated at $80 \mathrm{mK}$. The SQUID and detector bias lines are carried from the detector PCBs on twisted pair NbTi cables to the SA boards which are housed on the $1 \mathrm{~K}$ stage.

Each detector wafer was assembled with its respective read-out $\mathrm{PCB}(\mathrm{s})$ and tested individually prior to assembly onto the full array structure. While the final configuration of the PCB relative to the wafer is at 90 degrees as shown in Figure 2, the wire-bonding connecting the two must be performed in a flat configuration. An assembly jig was developed for the constrained and repeatable rotation of the PCB after attachment to the detector wafer. After folding, each wafer and readout assembly was lifted and placed onto the feedhorn array using a vacuum jig. Precision placement onto the alignment pins was achieved with microscope-coupled cameras.

The feed horn stack itself is mounted to the copper array structure using a set of six flexible copper L-brackets, designed to accommodate the differential thermal contraction of the copper and silicon. An image of the fully assembled PA1 array can be seen in Figure 2.

\subsection{Yield}

While the fabrication yield of the detector wafers was high (typically only $0-2$ non-continuous TESes per wafer), the assembly process of these monolithic wafers presents a number of challenges for maximizing the achieved read-out yield. Each assembled wafer, flex, and circuit board(s) functions as a single unit, making the correction of individual detector lines problematic. The largest detector losses in PA1 come from the loss of SQUID biasing and readout lines, which take out an entire row or column corresponding to 32 TESes. The flexible circuitry was also identified as a particular source of detector loss for PA1, both through open traces and difficulty of wire bonding to the soft flex substrate. The main sources of detector loss in the system include open SQ1 bias lines (13\% loss), non-working SQ2s (9\% loss), individual detector opens (9\% loss), individual detector shorts (4\% loss), bias lines masked due to excess array heating ( $3 \%$ loss), and non-working SQ1s ( $2 \%$ loss). The experience gained through the assembly of PA1, as the first implementation of the complicated procedure, has lead to the development of new assembly protocols to minimize these losses. Laboratory characterization of PA2 achieved $80 \%$ readout yield, with some wafers having as high as $92 \%$ yield.

\section{OBSERVING}

In the first season of observations, ACTPol observed four patches which we call D1, D2, D5, and D6, each covering $\sim 70$ square degrees and located approximately on the celestial equator. These fields were selected to overlap with a range of multi-frequency surveys. For each patch, the telescope scans in azimuth at a rate of $1.5 \mathrm{deg} / \mathrm{s}$ at a series of fixed elevations. The primary data acquisition file is the time-ordered data file or TOD, each of which contains approximately 10 minutes of data. The telescope steps in elevation after a period of scanning to follow the observed field as it rises in the east or sets in the west.

Due to the uninterrupted cooling provided by the dilution refrigerator, ACTPol was able to observe continuously through both day and night. Analyzing the daytime data requires a detailed understanding of the changing

Proc. of SPIE Vol. $9153915310-5$ 


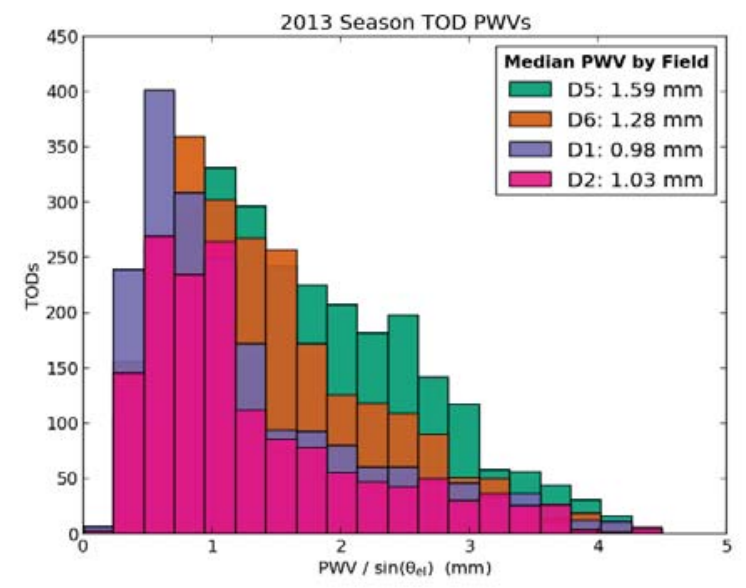

Figure 3: The observed 2013 season scaled PWVs $\left(\mathrm{PWV} / \sin \left(\theta_{e l}\right)\right)$ for all TODs for the four primary observation fields. This is the most significant driver of optical loading variation for the detectors. The 2013 season saw atypically high PWV conditions.

shape of the beam due to the distortion of the mirrors as they heat unevenly throughout the day. While the most recent analysis ${ }^{6}$ does not include the day-time data for the main power-spectra or parameter estimation, future releases will incorporate it.

The quality of observing is partly driven by the precipitable water content (PWV) of the atmosphere through its effect on the optical loading seen by the detectors (see Section 5). The quantity of interest in determining the loading is the PWV scaled by the airmass through the atmosphere, PWV $/ \sin \left(\theta_{e l}\right)$ or "scaled PWV". The median scaled PWV values as measured by the nearby APEX radiometer ${ }^{19}$ for fields D1, D2, D5, and D6 were $0.98,1.03,1.59$, and $1.28 \mathrm{~mm}$ respectively with the distributions shown in Figure 3. This is higher than other ACT observation years with 2008-2010 having a median scaled PWV of $0.9 \mathrm{~mm}$. A cut was used to exclude data taken in conditions of greater $3 \mathrm{~mm}$ PWV.

\section{CALIBRATION}

The ACTPol observing routine includes regular calibration and characterization measurements of the detectors. This calibration program involves point source measurements, taken every other night, detector bias ramps, taken at the beginning of observing each field, and detector bias steps, taken at every elevation change.

Planet scans are used to track the beam profiles and detector pointing. From these measurements the FWHM is determined as $1.36^{\prime}\left(1.26^{\prime}\right)$ along the major (minor) axis. ${ }^{6}$ The detector bias ramp (IV curve) is used to both establish the calibration of the raw TES data into absolute units and to determine the optimal detector bias under the observed loading conditions. The bias step measurement, in which a square wave is input onto the TES bias line, functions as a two point load curve allowing the extraction of calibration information. The bias step is also used to monitor variations in the detector time constants as will be discussed in Section 6 .

Several optimizations were made to the calibration strategy throughout the season. While the bias step and the IV curve can both be used to measure the detector responsivity, or current-to-power calibration, only the IV curve can be used to select the optimal bias point by exploring the full range of the TES transition. However, the IV curve has the disadvantage of causing heating of the array package because large biases are necessary to drive the TESes normal. Because of this heating, 220 seconds of data were cut after each IV acquisition. An optimal frequency of IV curve acquisitions was determined to allow rebiasing in response to loading changes while limiting the observed heating. The thermal impact of the IV curves was also minimized by reducing the pause time at each bias point from $100 \mathrm{~ms}$ to $25 \mathrm{~ms}$. This was found to cause no degradation to the ability to select detector bias currents or to measure calibration parameters. The frequency of bias step measurements was increased later in the season to allowing tracking of the detector responsivity between IV rebiasing events. 

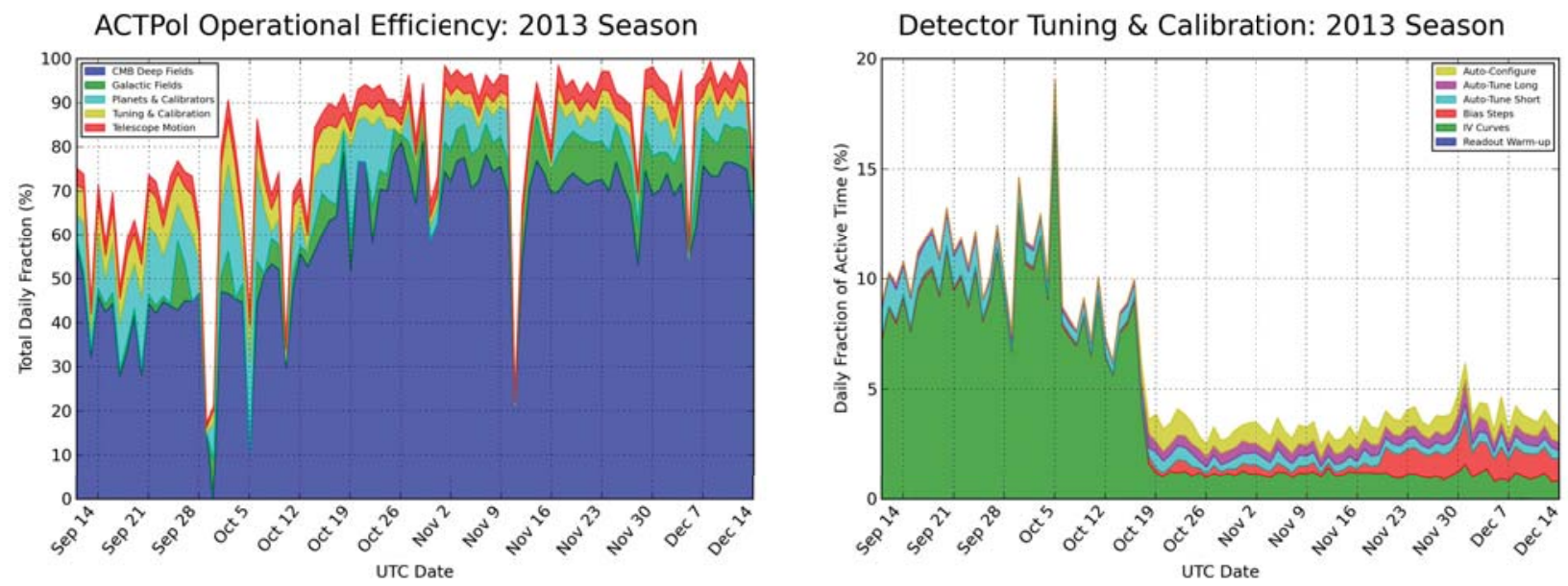

Figure 4: a) The ACTPol observing efficiency improved throughout the 2013 season with less than $10 \%$ daily idle time achieved throughout the second half of the season. One hour each day is dedicated to checks of the telescope systems at the site. b) Optimizations were made throughout the season to the observing and calibration schedules, including changes to the IV curve and bias step acquisitions. The calibration measurements take up less than $5 \%$ of active telescope time in the final calibration schedule.

Because each detector bias line is used for many detectors in series (96 TESes for a semihex and 64 for a hex), successful simultaneous biasing of the TESes requires a high degree of uniformity of their thermal properties. The biasing algorithm targets a particular bias point $\left(\mathrm{R} / \mathrm{R}_{N}\right)$ and then attempts to maximize the number of working detectors near this point. Given some non-zero spread in the thermal properties, attempting to bias too close to either edge of the transition causes some TESes to go superconducting or normal. The target bias point was selected as $40 \%$ of the normal resistance $\left(R_{n}\right)$ to optimize the achieved yield.

The season observing efficiency including the time spent on calibration, tuning, and biasing can be seen in Figure 4.

\section{LOADING}

Minimizing background loading on the detectors is key to optimizing their sensitivity. The optical loading on the detectors is dominated by atmospheric emission and loading from optical components of the telescope itself. The atmospheric loading can be further separated into a component from water vapor emission, which varies with PWV, and a component due to emission from other atmospheric constituents. The effects of the three components can be extracted by observing their differing dependencies on the PWV and observing elevation.

$$
P_{\text {bias }}=P_{\text {sat }}-\eta_{\text {det }}\left(P_{\text {optics }}+\frac{C_{\mathrm{wet}} P W V}{\sin \left(\theta_{e l}\right)}+\frac{C_{\mathrm{dry}}}{\sin \left(\theta_{e l}\right)}\right)
$$

Here $P_{\text {optics }}, C_{\text {wet }}$, and $C_{\mathrm{dry}}$ are fit coefficients describing the relative loading contributions, defined at the sky side of the feedhorn aperture, of the telescope optics, the wet atmosphere, and the dry atmosphere and $\eta_{\text {det }}$ is the optical coupling efficiency of each detector as defined in Table 1. An example fit for one detector can be seen in Figure 5. In performing this fit on the early season data, we found that the $P_{\text {optics }}$ term is larger than expectation. The addition of a reflective, half-conical forebaffle outside the window aperture was found to significantly reduce this excess. This improvement can be understood as the sidelobes of the beams being reflected to cold sky rather than observing the warm telescope components. The median reduction in loading for hex and semihex pixels was $1.2 \mathrm{pW}$ and $0.6 \mathrm{pW}$ respectively, where the difference arises due to the higher optical coupling efficiency of the hex pixels. In the final configuration, we find $P_{\text {optics }}=2.5 \pm 1.0 \mathrm{pW}$, $C_{\text {wet }}=2.3 \pm 0.4 \mathrm{pW} / \mathrm{mm}$, and $C_{\mathrm{dry}}=1.6 \pm 0.3 \mathrm{pW}$, where the error bars are the standard deviations of the detector distributions. 

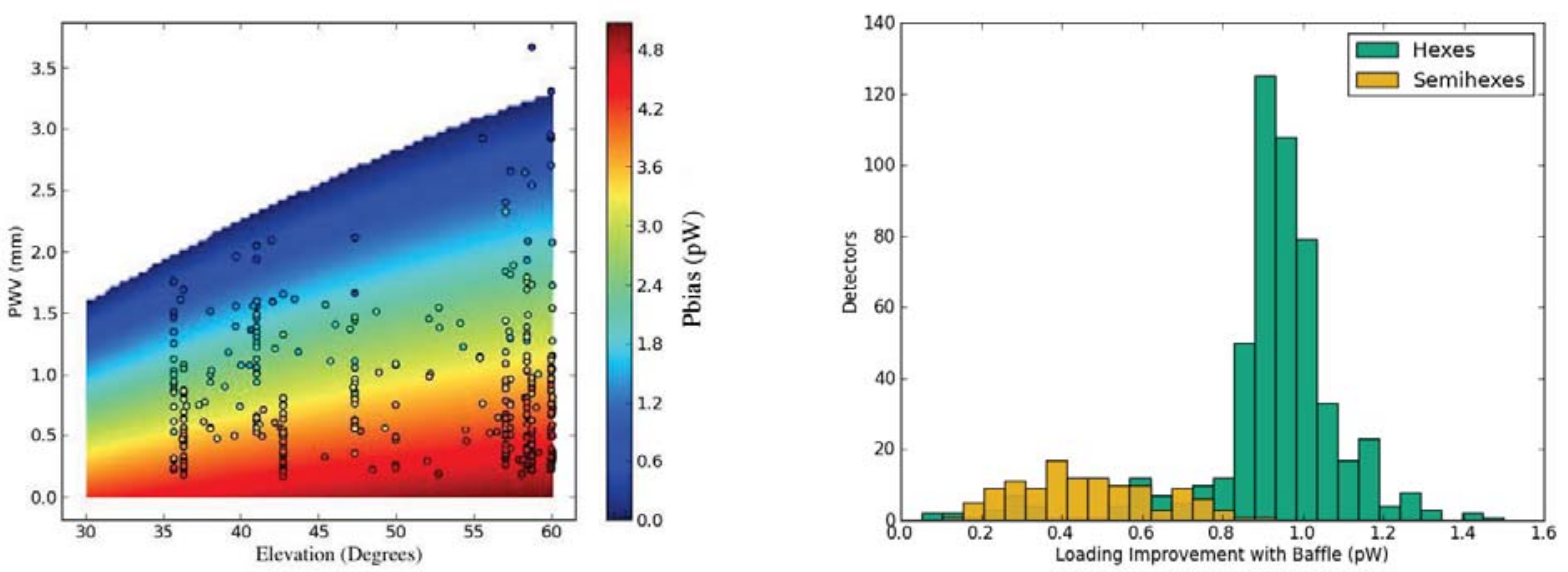

Figure 5: (Left) An example of the fit for the dependence of an individual detector's saturation power on the PWV and telescope pointing, the dominate sources of loading variation. Here the data points are from individual IV curves throughout the season and are colored based on their measured saturation powers. The background gradient shows the fit result. The fit separates the loading into three sources - constant background loading from the telescope optics, atmospheric loading due to water vapor, and other atmospheric loading. This fit shows the range of observing conditions under which the detector is operational. (Right) The reduction in the observed loading due to the installation of a half-conical baffle at the receiver window. This baffle mitigates the side lobe response to the warm telescope optics. The difference in improvement between the hexes and the semihexes is explained by their relative optical coupling efficiencies.

\section{TIME CONSTANTS}

The detector response at high frequencies is diminished by the electro-thermal time constant of the TESes. Measuring the detector time constants is key to accounting for this filtering effect in the analysis.

The intrinsic thermal time constant of a TES is determined by its heat capacity $(C)$ and its thermal conductivity $(G)$ to the bath. ${ }^{20}$

$$
\tau=\frac{C}{G}
$$

Given that the target thermal conductivity is optimized for noise considerations, the desired time constant is tuned by varying the heat capacity of the TES island through the addition of excess heat capacity in the form of a layer of PdAu. In its voltage biased configuration, the response of the TES is sped up through the negative electrothermal feedback. The detector time constants are therefore dependent on the biasing conditions of the TES. ${ }^{21}$ Expressed as an $f_{3 \mathrm{~dB}}$ (or frequency with $50 \%$ of the response), the dependence is:

$$
f_{3 \mathrm{~dB}}=\frac{1}{2 \pi \tau}=\frac{1}{2 \pi}\left(\frac{G}{C}+\frac{\alpha}{1+\beta} \frac{1}{T_{c} C} P_{\text {bias }}\right) .
$$

Here $P_{\text {bias }}$ is the Joule power applied on the TES through the TES bias line and $\alpha=\frac{T}{R} \frac{d R}{d T}$ and $\beta=\frac{I}{R} \frac{d R}{d I}$ are the logarithmic derivatives with respect to temperature and current of the detector's resistance. These parameters are used to characterize the transition shape.

For ACTPol, the two primary methods used to calibrate the detector time constants are planet scans and bias steps. When a point source is scanned from two opposite scan directions, a finite time constant causes a shift in the apparent source peak position. The bias step method measures the settling time of the TES current after each step in the square wave input by fitting the response to an exponential rolloff, $I \sim e^{\frac{-t}{\tau}}$. 

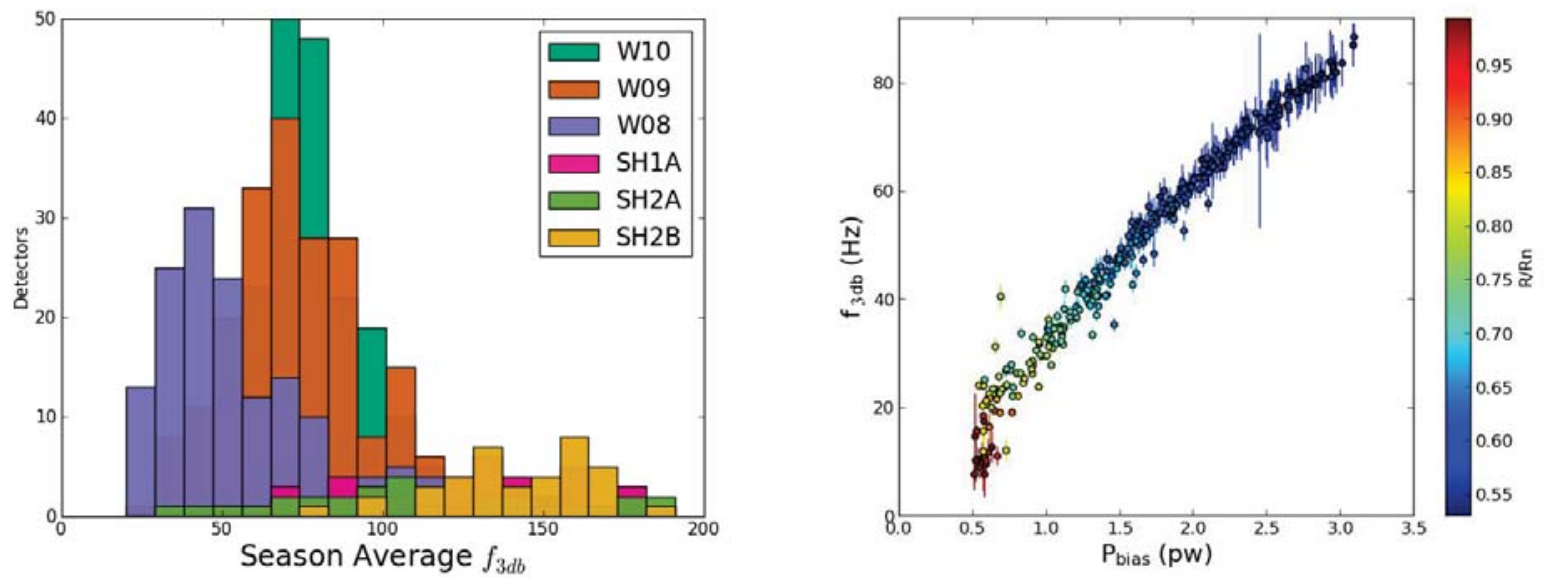

Figure 6: (Left) The season median observed $f_{3 \mathrm{~dB}} \mathrm{~s}$. The differences between wafers are driven by variation in the TES thermal properties, including the saturation powers which determine the bias powers. (Right) An example for an individual detector of the $f_{3 \mathrm{~dB}}$ variation seen across the 2013 season. At a constant bias point on the transition, the $f_{3 \mathrm{~dB}}$ should depend linearly with the bias power. Changes in the slope of this relationship can occur as the bias point varies. The strength of the electrothermal feedback is decreased on the edges of the transition, slowing the time constants in these regions. Because the TES bias is chosen consistently at a given loading, the bias point and bias power vary predictably together, allowing the $f_{3 \mathrm{~dB}}$ to be modeled as a function of bias power alone.

Significant variation of the detector time constants is observed under different loading conditions. This is driven primarily by changes in the bias power. Additionally, the $\alpha$ and $\beta$ dependence causes variation with the TES bias point $\left(R / R_{N}\right)$. An example of the $P_{\text {bias }}$ and $R / R_{N}$ dependence for a particular detector can be seen in Figure 6. This relationship can be understood from Equation 5 as the $f_{3 \mathrm{~dB}}$ varying linearly with $P_{\text {bias }}$. The slope can vary with bias point, particularly high or low on the transition where the transition shape changes significantly. Given that the chosen bias point follows a predictable trajectory with loading, the $f_{3 \mathrm{~dB}} \mathrm{c}$ can be fully modeled as a function of $P_{\text {bias }}$ alone.

Because the planet scan method and the bias step method for time constant measurement rely on different mechanisms for inputting power onto the TES, there is potential for differing thermal paths to cause a discrepancy between the two methods. The planet scans, which operate using the same optical coupling as CMB observations, are taken to be the standard. A comparison finds that the bias steps results give $\sim 16 \%$ higher $f_{3 \mathrm{~dB}} \mathrm{~s}$. A conversion factor is applied to the bias step results to account for this.

To correct the detector time streams for the filtering of the time constant, each detector was assigned a time-dependent $f_{3 \mathrm{~dB}}$ for each TOD. The method of assigning this value varied based on the set of calibration measurements available. When a recent bias step acquisition had been taken, the bias step result (scaled using the bias step to planet scan conversion factor) was used directly. However, the bias step was not a frequent part of the calibration process early in the season. For these TODs, as long as a recent measurement of the detector bias powers was available from an IV, the model of $f_{3 \mathrm{~dB}}$ versus $P_{\text {bias }}$ was used. Finally, without a recent IV available, the bias power was predicted from the observed PWV before use in the $f_{3 \mathrm{~dB}}$ versus $P_{\text {bias }}$ model.

Across all TODS, the median $f_{3 \mathrm{~dB}}$ for the full season was $70 \mathrm{~Hz}$. The distribution of the season average $f_{3 \mathrm{~dB}}$ results can be seen in Figure 6. Detectors with $f_{3 \mathrm{~dB}}<20 \mathrm{~Hz}$ are cut from the analysis. The typical dependence on the scaled PWV was an $f_{3 \mathrm{~dB}}$ decrease of $17 \mathrm{~Hz} / \mathrm{mm}$.

\section{NOISE}

The noise-equivalent temperatures (NETs) of the ACTPol detectors have been estimated using two methods, one based on the time-stream noise at $20 \mathrm{~Hz}$ and the other based on pixel noise in map space. The map-based 


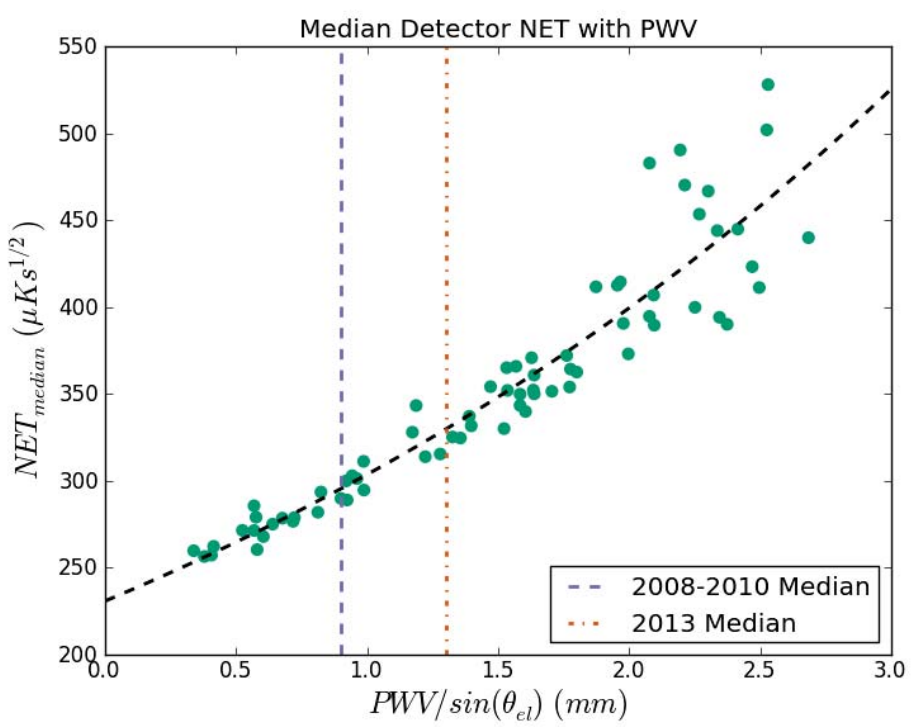

Figure 7: The median detector sensitivity as a function of the scaled PWV estimated from individual time streams. The dashed line is an empirical, exponential fit to find the general trend. The median scaled PWV for the 2013 season was $1.3 \mathrm{~mm}$, higher than the typical value of $0.9 \mathrm{~mm}$ observed during the 2008-2011 ACT seasons. Around $5 \%$ of this difference is explained by a different median observing elevation, but the majority of the increase is caused by the atypical weather.

estimate gives a median detector NET of $340 \mu \mathrm{K} \sqrt{\mathrm{s}}$.

Because the ACTPol detectors are photon-noise limited, the total array sensitivity is dependent on the amount of optical loading. Using NETs estimated from individual TODs, the level of this effect can be determined. The variation of median detector sensitivity can be seen in Figure 7. The decrease in sensitivity with loading is driven both by the increased photon noise and the saturation of detectors. Extrapolating to zero PWV results in a median detector sensitivity of $230 \mu \mathrm{K} \sqrt{\mathrm{s}}$. Using the distribution of observed scaled PWVs with a season median of $1.3 \mathrm{~mm}$, the effective detector median NET is $330 \mu \mathrm{K} \sqrt{\mathrm{s}}$, consistent with the map-based noise estimate. This result shows that an improvement in the sensitivity can be expected if PWV levels return to more typical values for future observation seasons.

In the three months of observing time in the first season a total of 1030 hours of data were used produce the maps of the initial data release. The achieved white-noise map sensitivities in each of the four observation fields D1, D2, D5, and D6 were 16.2, 17, 13.2, and $11.2 \mu \mathrm{K}$-arcmin respectively.

\section{CONCLUSIONS}

The first season of ACTPol observations, consisting of three months of data with only a subset of the total planned deployment detectors, has demonstrated the capabilities of the new receiver with an improvement in mapping speed of more than three times that of the original ACT receiver. The deployment of two additional detector arrays will provide substantial improvements in sensitivity.

\section{ACKNOWLEDGMENTS}

This work was supported by the U.S. National Science Foundation through awards AST-0408698 and AST0965625 for the ACT project, as well as awards PHY-0855887 and PHY-1214379. Funding was also provided by Princeton University, the University of Pennsylvania, and a Canada Foundation for Innovation (CFI) award to UBC. ACT operates in the Parque Astronómico Atacama in northern Chile under the auspices of the Comisión Nacional de Investigación Científica y Tecnológica de Chile (CONICYT). Computations were performed on the GPC supercomputer at the SciNet HPC Consortium. SciNet is funded by the CFI under the auspices of Compute 
Canada, the Government of Ontario, the Ontario Research Fund - Research Excellence; and the University of Toronto. Funding from ERC grant 259505 supports SN. BS, BK, CM, and EG are funded by NASA Space Technology Research Fellowships. We thank our many colleagues from ABS, ALMA, APEX, and POLARBEAR who have helped us at critical junctures. We thank Masao Uehara, our site engineer, who kept ACT operational day in and day out. Colleagues at AstroNorte and RadioSky provide logistical support and keep operations in Chile running smoothly. We also thank the Mishrahi Fund and the Wilkinson Fund for their generous support of the project.

\section{REFERENCES}

[1] Sievers, J. L., Hlozek, R. A., Nolta, M. R., Acquaviva, V., Addison, G. E., Ade, P. A. R., Aguirre, P., Amiri, M., Appel, J. W., Barrientos, L. F., Battistelli, E. S., Battaglia, N., Bond, J. R., Brown, B., Burger, B., Calabrese, E., Chervenak, J., Crichton, D., Das, Sudeep, Devlin, M. J., Dicker, S. R., Bertrand Doriese, W., Dunkley, J., Dnner, R., Essinger-Hileman, T., Faber, D., Fisher, R. P., Fowler, J. W., Gallardo, P., Gordon, M. S., Gralla, M. B., Hajian, A., Halpern, M., Hasselfield, M., Hernndez-Monteagudo, C., Hill, J. C., Hilton, G. C., Hilton, M., Hincks, A. D., Holtz, D., Huffenberger, K. M., Hughes, D. H., Hughes, J. P., Infante, Leopoldo, Irwin, Kent D., Jacobson, David R., Johnstone, Brittany, Baptiste Juin, Jean, Kaul, Madhuri, Klein, Jeff, Kosowsky, Arthur, Lau, Judy M., Limon, M., Lin, Y., Louis, T., Lupton, R. H., Marriage, T. A., Marsden, D., Martocci, K., Mauskopf, P., McLaren, M., Menanteau, F., Moodley, K., Moseley, H., Netterfield, C. B., Niemack, M. D., Page, L. A., Page, W. A., Parker, L., Partridge, B., Plimpton, R., Quintana, H., Reese, E. D., Reid, B., Rojas, F., Sehgal, Neelima, Sherwin, Blake D., Schmitt, Benjamin L., Spergel, David N., Staggs, Suzanne T., Stryzak, Omelan, Swetz, Daniel S., Switzer, Eric R., Thornton, R., Trac, H., Tucker, C., Uehara, M., Visnjic, K., Warne, R., Wilson, G., Wollack, E., Zhao, Y., and Zunckel, C. "The Atacama Cosmology Telescope: Cosmological parameters from three seasons of data," JCAP 10, 60 (2013).

[2] Planck Collaboration, Ade, P. A. R., Aghanim, N., Alves, M. I. R., Armitage-Caplan, C., Arnaud, M., Ashdown, M., Atrio-Barandela, F., Aumont, J., Aussel, H., Baccigalupi, C., Banday, A. J., Barreiro, R. B., Barrena, R., Bartelmann, M., Bartlett, J. G., Bartolo, N., Basak, S., Battaner, E., Battye, R., Benabed, K., Benot, A., Benoit-Lvy, A., Bernard, J.-P., Bersanelli, M., Bertincourt, B., Bethermin, M., Bielewicz, P., Bikmaev, I., Blanchard, A., Bobin, J., Bock, J. J., Bhringer, H., Bonaldi, A., Bonavera, L., Bond, J. R., Borrill, J., Bouchet, F. R., Boulanger, F., Bourdin, H., Bowyer, J. W., Bridges, M., Brown, M. L., Bucher, M., Burenin, R., Burigana, C., Butler, R. C., Calabrese, E., Cappellini, B., Cardoso, J.-F., Carr, R., Carvalho, P., Casale, M., Castex, G., Catalano, A., Challinor, A., Chamballu, A., Chary, R.-R., Chen, X., Chiang, H. C., Chiang, L.-Y, Chon, G., Christensen, P. R., Churazov, E., Church, S., Clemens, M., Clements, D. L., Colombi, S., Colombo, L. P. L., Combet, C., Comis, B., Couchot, F., Coulais, A., Crill, B. P., Cruz, M., Curto, A., Cuttaia, F., Da Silva, A., Dahle, H., Danese, L., Davies, R. D., Davis, R. J., de Bernardis, P., de Rosa, A., de Zotti, G., Dchelette, T., Delabrouille, J., Delouis, J.-M., Dmocls, J., Dsert, F.-X., Dick, J., Dickinson, C., Diego, J. M., Dolag, K., Dole, H., Donzelli, S., Dor, O., Douspis, M., Ducout, A., Dunkley, J., Dupac, X., Efstathiou, G., Elsner, F., Enlin, T. A., Eriksen, H. K., Fabre, O., Falgarone, E., Falvella, M. C., Fantaye, Y., Fergusson, J., Filliard, C., Finelli, F., Flores-Cacho, I., Foley, S., Forni, O., Fosalba, P., Frailis, M., Fraisse, A. A., Franceschi, E., Freschi, M., Fromenteau, S., Frommert, M., Gaier, T. C., Galeotta, S., Gallegos, J., Galli, S., Gandolfo, B., Ganga, K., Gauthier, C., Gnova-Santos, R. T., Ghosh, T., Giard, M., Giardino, G., Gilfanov, M., Girard, D., Giraud-Hraud, Y., Gjerlw, E., Gonzlez-Nuevo, J., Grski, K. M., Gratton, S., Gregorio, A., Gruppuso, A., Gudmundsson, J. E., Haissinski, J., Hamann, J., Hansen, F. K., Hansen, M., Hanson, D., Harrison, D. L., Heavens, A., Helou, G., Hempel, A., Henrot-Versill, S., Hernndez-Monteagudo, C., Herranz, D., Hildebrandt, S. R., Hivon, E., Ho, S., Hobson, M., Holmes, W. A., Hornstrup, A., Hou, Z., Hovest, W., Huey, G., Huffenberger, K. M., Hurier, G., Ili?, S., Jaffe, A. H., Jaffe, T. R., Jasche, J., Jewell, J., Jones, W. C., Juvela, M., Kalberla, P., Kangaslahti, P., Keihnen, E., Kerp, J., Keskitalo, R., Khamitov, I., Kiiveri, K., Kim, J., Kisner, T. S., Kneissl, R., Knoche, J., Knox, L., Kunz, M., Kurki-Suonio, H., Lacasa, F., Lagache, G., Lhteenmki, A., Lamarre, J.-M., Langer, M., Lasenby, A., Lattanzi, M., Laureijs, R. J., Lavabre, A., Lawrence, C. R., Le Jeune, M., Leach, S., Leahy, J. P., Leonardi, R., Len-Tavares, J., Leroy, C., Lesgourgues, J., Lewis, A., Li, C., Liddle, A., Liguori, M., Lilje, P. B., Linden-Vrnle, M., Lindholm, V., Lpez-Caniego, M., Lowe, S., Lubin, 
P. M., Macas-Prez, J. F., MacTavish, C. J., Maffei, B., Maggio, G., Maino, D., Mandolesi, N., Mangilli, A., Marcos-Caballero, A., Marinucci, D., Maris, M., Marleau, F., Marshall, D. J., Martin, P. G., MartnezGonzlez, E., Masi, S., Massardi, M., Matarrese, S., Matsumura, T., Matthai, F., Maurin, L., Mazzotta, P., McDonald, A., McEwen, J. D., McGehee, P., Mei, S., Meinhold, P. R., Melchiorri, A., Melin, J.-B., Mendes, L., Menegoni, E., Mennella, A., Migliaccio, M., Mikkelsen, K., Millea, M., Miniscalco, R., Mitra, S., Miville-Deschnes, M.-A., Molinari, D., Moneti, A., Montier, L., Morgante, G., Morisset, N., Mortlock, D., Moss, A., Munshi, D., Murphy, J. A., Naselsky, P., Nati, F., Natoli, P., Negrello, M., Nesvadba, N. P. H., Netterfield, C. B., Nrgaard-Nielsen, H. U., North, C., Noviello, F., Novikov, D., Novikov, I., O’Dwyer, I. J., Orieux, F., Osborne, S., O’Sullivan, C., Oxborrow, C. A., Paci, F., Pagano, L., Pajot, F., Paladini, R., Pandolfi, S., Paoletti, D., Partridge, B., Pasian, F., Patanchon, G., Paykari, P., Pearson, D., Pearson, T. J., Peel, M., Peiris, H. V., Perdereau, O., Perotto, L., Perrotta, F., Pettorino, V., Piacentini, F., Piat, M., Pierpaoli, E., Pietrobon, D., Plaszczynski, S., Platania, P., Pogosyan, D., Pointecouteau, E., Polenta, G., Ponthieu, N., Popa, L., Poutanen, T., Pratt, G. W., Przeau, G., Prunet, S., Puget, J.-L., Pullen, A. R., Rachen, J. P., Racine, B., Rahlin, A., Rth, C., Reach, W. T., Rebolo, R., Reinecke, M., Remazeilles, M., Renault, C., Renzi, A., Riazuelo, A., Ricciardi, S., Riller, T., Ringeval, C., Ristorcelli, I., Robbers, G., Rocha, G., Roman, M., Rosset, C., Rossetti, M., Roudier, G., Rowan-Robinson, M., Rubio-Martn, J. A., Ruiz-Granados, B., Rusholme, B., Salerno, E., Sandri, M., Sanselme, L., Santos, D., Savelainen, M., Savini, G., Schaefer, B. M., Schiavon, F., Scott, D., Seiffert, M. D., Serra, P., Shellard, E. P. S., Smith, K., Smoot, G. F., Souradeep, T., Spencer, L. D., Starck, J.-L., Stolyarov, V., Stompor, R., Sudiwala, R., Sunyaev, R., Sureau, F., Sutter, P., Sutton, D., Suur-Uski, A.-S., Sygnet, J.-F., Tauber, J. A., Tavagnacco, D., Taylor, D., Terenzi, L., Texier, D., Toffolatti, L., Tomasi, M., Torre, J.-P., Tristram, M., Tucci, M., Tuovinen, J., Trler, M., Tuttlebee, M., Umana, G., Valenziano, L., Valiviita, J., Van Tent, B., Varis, J., Vibert, L., Viel, M., Vielva, P., Villa, F., Vittorio, N., Wade, L. A., Wandelt, B. D., Watson, C., Watson, R., Wehus, I. K., Welikala, N., Weller, J., White, M., White, S. D. M., Wilkinson, A., Winkel, B., Xia, J.-Q., Yvon, D., Zacchei, A., Zibin, J. P., and Zonca, A. "Planck 2013 results. I. Overview of products and scientific results," arxiv:1303.5062 (2013)

[3] Hou, Z., Reichardt, C. L., Story, K. T., Follin, B., Keisler, R., Aird, K. A., Benson, B. A., Bleem, L. E., Carlstrom, J. E., Chang, C. L., Cho, H.-M., Crawford, T. M., Crites, A. T., de Haan, T., de Putter, R., Dobbs, M. A., Dodelson, S., Dudley, J., George, E. M., Halverson, N. W., Holder, G. P., Holzapfel, W. L., Hoover, S., Hrubes, J. D., Joy, M., Knox, L., Lee, A. T., Leitch, E. M., Lueker, M., Luong-Van, D., McMahon, J. J., Mehl, J., Meyer, S. S., Millea, M., Mohr, J. J., Montroy, T. E., Padin, S., Plagge, T., Pryke, C., Ruhl, J. E., Sayre, J. T., Schaffer, K. K., Shaw, L., Shirokoff, E., Spieler, H. G., Staniszewski, Z., Stark, A. A., van Engelen, A., Vanderlinde, K., Vieira, J. D., Williamson, R., and Zahn, O., "Constraints on Cosmology from the Cosmic Microwave Background Power Spectrum of the $2500 \operatorname{deg}^{2}$ SPT-SZ Survey," ApJ, 782, 74 (2014).

[4] Hinshaw, G., Larson, D., Komatsu, E., Spergel, D. N., Bennett, C. L., Dunkley, J., Nolta, M. R., Halpern, M., Hill, R. S., Odegard, N., Page, L., Smith, K. M., Weiland, J. L., Gold, B., Jarosik, N., Kogut, A., Limon, M., Meyer, S. S., Tucker, G. S., Wollack, E., and Wright, E. L. "Nine-year Wilkinson Microwave Anisotropy Probe (WMAP) Observations: Cosmological Parameter Results," ApJS 208, 19-25 (2013).

[5] Balbi, A., Natoli, P., and Vittorio, N., "The CMB polarization: status and prospects," arxiv:astro$\mathrm{ph} / 0606511(2006)$.

[6] Naess, S., Hasselfield, M., McMahon, J., Niemack, M. D., Addison, G. E., Ade, P. A. R., Allison, R., Amiri, M., Baker, A., Battaglia, N., Beall, J. A., de Bernardis, F., Bond, J R., Britton, J., Calabrese, E., Cho, H. M., Coughlin, K., Crichton, D., Das, S., Datta, R., Devlin, M. J., Dicker, S. R., Dunkley, J., Dnner, R., Fowler, J. W., Fox, A. E., Gallardo, P., Grace, E., Gralla, M., Hajian, A., Halpern, M., Henderson, S., Hill, J. C., Hilton, G. C., Hilton, M., Hincks, A. D., Hlozek, R., Ho, P., Hubmayr, J., Huffenberger, K. M., Hughes, J. P., Infante, L., Irwin, K., Jackson, R., Klein, J., Koopman, B., Kosowsky, A., Li, D., Louis, T., Lungu, M., Madhavacheril, M., Marriage, T. A., Maurin, L., Menanteau, F., Moodley, K., Munson, C., Newburgh, L., Nibarger, J., Nolta, M. R., Page, L. A., Pappas, C., Partridge, Bruce, Rojas, Felipe, Schmitt, Benjamin, Sehgal, Neelima, Sherwin, Blake D., Sievers, Jon, Simon, Sara, Spergel, David N., Staggs, Suzanne T., Switzer, E. R., Thornton, R., Trac, H., Tucker, C., Van Engelen, A., Ward, and J., Wollack, E. J., "The Atacama Cosmology Telescope: CMB Polarization at $200<\ell<9000$," arxiv:1405.5524 (2014). 
[7] Fowler, J. W., Niemack, M. D., Dicker, S. R., Aboobaker, A. M., Ade, P. A. R., Battistelli, E. S., Devlin, M. J., Fisher, R. P., Halpern, M., Hargrave, P. C., Hincks, A. D., Kaul, M., Klein, J., Lau, J. M., Limon, M., Marriage, T. A., Mauskopf, P. D., Page, L., Staggs, S. T., Swetz, D. S., Switzer, E. R., Thornton, R. J., and Tucker, C. E., "Optical design of the Atacama Cosmology Telescope and the Millimeter Bolometric Array Camera," Appl. Opt. 46(17), 34443454 (2007).

[8] Niemack, M. D., Ade, P. A. R., Aguirre, J., Barrientos, F., Beall, J. A., Bond, J. R., Britton, J., Cho, H. M., Das, S., Devlin, M. J., Dicker, S., Dunkley, J., Dnner, R., Fowler, J. W., Hajian, A., Halpern, M., Hasselfield, M., Hilton, G. C., Hilton, M., Hubmayr, J., Hughes, J. P., Infante, L., Irwin, K. D., Jarosik, N., Klein, J., Kosowsky, A., Marriage, T. A., McMahon, J., Menanteau, F., Moodley, K., Nibarger, J. P., Nolta, M. R., Page, L. A., Partridge, B., Reese, E. D., Sievers, J., Spergel, D. N., Staggs, S. T., Thornton, R., Tucker, C., Wollack, E., and Yoon, K. W. "ACTPol: A polarization-sensitive receiver for the Atacama Cosmology Telescope," Proc. SPIE 774, (2010)

[9] Datta, R., Munson, C. D., Niemack, M. D., McMahon, J. J., Britton, J., Wollack, E. J., Beall, J., Devlin, M. J., Fowler, J., Gallardo, P., Hubmayr, J., Irwin, K., Newburgh, L., Nibarger, J. P., Page, L., Quijada, M. A., Schmitt, B.L, Staggs, S. T., Thornton, R., and Zhang, L. "Large-aperture wide-bandwidth antireection-coated silicon lenses for millimeter wavelengths," Appl. Opt. 52 (36), 8747-8758 (2013).

[10] Datta, R., Hubmayr, J., Munson, C., Austermann, J., Beall, J., Becker, D., Cho, H. M., Halverson, N., Hilton, G., Irwin, K., Li, D., McMahon, J., Newburgh, L., Nibarger, J., Niemack, M., Schmitt, B., Smith, H., Staggs, S., Van Lanen, J., and Wollack, E. "Horn Coupled Multichroic Polarimeters for the Atacama Cosmology Telescope Polarization Experiment," J. Low Temp. Phys. 176, (2014).

[11] Britton, J. W., Nibarger, J. P, Yoon, K. W., Beall, J. A., Becker, D., Cho, H. M., Hilton, G. C., Hubmayr, J., Niemack, M. D., and Irwin, K. D. "Corrugated Silicon Platelet Feed Horn Array for CMB Polarimetry at $150 \mathrm{GHz}, "$ Proc. SPIE 774 (2010).

[12] Ade, P. A. R., Pisano, G., Tucker, C., and Weaver, S., "A review of metal mesh filters," Proc. SPIE 6275 (2006).

[13] Tucker, C.E and Ade, P.A.R., "Thermal filtering for large aperture cryogenic detector arrays", Proc. SPIE $6275(2006)$.

[14] Grace, E. A., Beall, J., Cho, H. M., Devlin, M. J., Fox, A., Hilton, G., Hubmayr, J., Irwin, K., Klein, J., Li, D., Lungu, M., Newburgh, L. B., Nibarger, J., Niemack, M. D., McMahon, J., Page, L. A., Pappas, C., Schmitt, B. L., Staggs, S. T., Van Lanen, J., and Wollack, E., "Characterization and Performance of a Kilo-TES Sub-Array for ACTPol," J. Low Temp. Phys. 176 (2014).

[15] Bock, J. J., Gundersen, J., Lee, A. T., Richards, P. L., and Wollack, E. J., "Optical coupling," J. Phys. Conference Series 155, 42-50 (2009).

[16] McMahon, J., Appel, J. W., Austermann, J. E., Beall, J. A., Becker, D., Benson, B. A., Bleem, L. E., Britton, J., Chang, C. L., Carlstrom, J. E., Cho, H. M., Crites, A. T., Essinger-Hileman, T., Everett, W., Halverson, N. W., Henning, J. W., Hilton, G. C., Irwin, K. D., Mehl, J., Meyer, S. S., Mossley, S., Niemack, M. D., Parker, L. P., Simon, S. M., Staggs, S. T., Visnjic, C., Wollack, E., U.-Yen, K., Yoon, K. W., and Zhao, Y. "Planar Orthomode Transducers for Feedhorn-coupled TES Polarimeters," Proc. LTD 1185, 162 (2009).

[17] Chervenak, J. A., Irwin, K. D., Grossman, E. N., Martinis, J. M., Reintsema, C. D., and Huber, M. E. "Superconducting multiplexer for arrays of transition edge sensors," Appl. Phys. Lett. 74 (26), 4043-4045 (1999).

[18] Battistelli, E. S., Amiri, M.; Burger, B., Halpern, M., Knotek, S., Ellis, M., Gao, X., Kelly, D.; Macintosh, M., Irwin, K., and Reintsema, C., "Functional description of read-out electronics for time-domain multiplexed bolometers for millimeter and sub-millimeter astronomy, " J. Low Temp. Phys. 151, 908914 (2008).

[19] Güsten, R., Nyman, L. Å., Schilke, P., Menten, K., Cesarsky, C., \& Booth, R., "The Atacama Pathnder EXperiment (APEX) a new submillimeter facility for southern skies ," A\&A 454, L13 (2006).

[20] Irwin,K. D. and Hilton, G. C. Transition Edge Sensors in Cryogenic Particle Detection, Springer-Verlag Berlin Heidelberg, 63-105 (2005).

[21] Niemack, M. D. Towards Dark Energy: Design, Development, and Preliminary Data from ACT, Ph.D. thesis, Princeton University (2008). 